\title{
Armas de fuego: ¿una respuesta a la inseguridad ciudadana? Su impacto y prevalencia en la morbilidad del AMSS2
}

\section{Introducción}

Las armas de fuego se han convertido en uno de los medios predilectos de los salvadoreños que han optado por resguardarse del clima violento y delincuencial transmitido diariamente por los medios de comunicación. De hecho, la seguridad de los ciudadanos salvadoreños se ha convertido en un gran negocio para las empresas que se encargan de vender armas y prestar servicios de seguridad privada. Es probable que los polígonos de tiro y las escuelas de manejo de armas se unan y complementen el negocio bélico de unos pocos.

La coyuntura actual ha puesto de manifiesto que no es necesario que exista un conflicto ammado para que una buena cantidad de armas se encuentre en circulación. A diferencia de la guerra civil salvadoreña, las armas no las empuñan ni las fuerzas gubernamentales ni las fuerzas insurgentes; ahora es un ejército constituido por cientos de personas civiles. No obstante, la década de los noventa, según datos de la Fiscalía General de la República, ha aportado más muertes, por año, que el mismo conflicto armado (González, 1997).

Según el discurso oficial y popular, el fenómeno delincuencial es el responsable de generar la atmósfera de violencia que se vive en el país. En ese mismo sentido algunos consideran que el problema de la violencia en América Latina está fuertemente asociado a la incidencia de hechos criminales (Rubio, 1998). Sin embargo, la violencia es eso y más. Querer igualar la violencia con la delincuencia es reducir e ideologizar un problema más amplio y complejo (IUDOP, 1996a; Echeburúa, 1996). En una visión como la anterior "se ignoran las responsabilidades propias en la prevalencia del fenómeno y se niegan las dinámicas que toleran y estimulan la delincuencia misma" (IUDOP, 1996a, p. 569). Es decir, la violencia puede venir tanto del ciudadano común como del delineuente.

En ese contexto también se encuentra la seguridad ciudadana para contrarrestar el efeclo de la violencia, el crimen y el sentimiento de vulnerabilidad de las 
personas. De hecho, la seguridad ciudadana hace referencia a dos fenómenos distintos pero vinculados: la criminalidad, entendida como todos aquellos hechos delictivos cometidos en una sociedad, y el sentimiento de inseguridad, es decir, la percepción de los ciudadanos sobre la criminalidad (IIDH, 1998). La seguridad ciudadana debe ser entendida como un compromiso tanto del Estado, específicamente de los cuerpos policiales y del sistema de justicia, como de los ciudadanos mismos para establecer un clima en el que se privilegien los derechos y la convivencia de la población en general. Sin embargo, la falta de confianza y credibilidad en las instituciones a las cuales les compete ofrecer seguridad ha permitido que esa cuota de responsabilidad sea asumida, en buena parte, por el ciudadano común.

Como consecuencia, muchas personas eslán dispuestas a actuar completamente fuera de la ley con el fin de obtener la justicia y la prolección que las instituciones gubermamentales no les brindan. Es acá donde la participación ciudadana, en el compromiso con su propia "seguridad", se convierte en una pieza más del mecanismo que legitima y mantiene con vida la violencia (IUDOP, 1998).

El presente artículo tiene como objetivo hacer una evaluación crítica de la eficacia de las amas de fuego como medio de protección y mostrar la incidencia y prevalencia de las armas de fuego en la morbilidad del Área Metropolitana de San Salvador (AMSS). Para ello se ha dividido el trabajo en cinco apartados. En el primer apartado se discute ampliamente el valor de las armas de fuego como instrumentos de protección personal. Con ese fin se citan diversos estudios y se aportan datos que respaldan o que ponen en entredicho la eficacia de ellas en materia de seguridad personal, incorporando integralmente ambas posturas al contexto salvadoreño. Además, se evalúa la relación entre armas de fuego, conducta agresiva y tasas de homicidios. En el segundo apartado, el autor trata de dar una aproximación cuantitativa y cualitativa de la dimensión del mercado y de la circulación de armas de fuego en el país, durante y después del conflicto armado. El tercer apartado incluye una serie de observaciones relacionadas a la ley de tenencia y portación de armas vigente. El cuarto apartado trata sobre la incidencia que tienen las armas de fuego en las causas de muerte reportadas para el AMSS, en los años 1995, 1996 y 1997, tomando como referencia registros del Inslituto de Medicina Legal y de las alcaldías del AMSS. Finalmente, en el quinto apartado se recogen una serie de reflexiones y conclusiones sobre el tema expuesto.

\section{Las armas de fuego como medio de protección personal}

A continuación se discutirá el valor de la portación y/o tenencia de una arma de fuego en la seguridad personal. Para ello, y con el objeto de hacerlo más comprensible, se ha dividido en tres puntos. En el primero se expondrán los argumentos a favor de las armas de fuego como instrumentos de protección 
personal. En el segundo se presentan, como contrapartida, los argumentos en contra de las armas. Finalmente, en el tercer punto, se hace una aproximación integral de ambas posturas. Es importante mencionar que aunque en los tres puntos se mencionan aspectos de la realidad del país relacionados con el problema en cuestión, el tercer apartado hace especial énfasis al contexto salvadoreño.

\subsection{Argumentos a favor}

Al parecer, el creciente flujo de información que los ciudadanos salvadoreños reciben a diario de los medios de comunicación sobre la delincuencia, ha exacerbado la apreciación de dicha problemática; esto sin subestimar la gravedad e importancia que merece. Frente a esla situación, la población considera que la seguridad, en primera instancia, tiene una relación proporcionalmente directa a las acciones encaminadas a procurársela a sí mismos. Según una investigación sobre viclimización ${ }^{3}$ realizada en el Área Metropolitana de San Salvador, el 8 por ciento de los ciudadanos de dicha área geográfica dijo haber adquirido un arma de fuego como medida de seguridad personal; mientras que otro porcentaje similar adquirió algún tipo de arma blanca como instrumento de defensa. Otro porcentaje de personas optó por relorzar las medidas de seguridad de su casa o contrató los servicios de agentes de seguridad privados.

En el caso de las personas que poseen y/o portan un arma, la razón más citada detrás de este comportamiento es la defensa propia. De hecho, según la última encuesta del IUDOP (1998), el 73 por ciento de las personas, en el ámbito nacional, que declararon tener algún arma de fuego, dicen poseerlas por razones de protección personal. Sin embargo, el término "protección personal" o "defensa propia" hay que manejarlo con mucho cuidado puesto que es vago (Roth, 1989), ya que depende de lo que las víctimas consideren como peligroso, o del momento o hecho ante el cual consideren necesario defenderse: frente a un disparo, una amenaza, un robo, etc.

Por tales razones, la atención no debe enfocarse únicamente en la letalidad del instrumento, sino en las características del usuario. Según distintos autores (Kates et al., 1994; Polsby y Brennen, 1995; Kellermann, 1996; Wintemute, 1998), los propietarios de armas con las cuales se perpetraron homicidios presentaban perfiles de comportamiento similares. Algunas características de dichos perfiles son: el consumo de alcohol, el uso ilícito de drogas, un historial previo de arresios y una historia de violencia familiar.

Además, resulta claro que el miedo experimentado puede motivar la adquisición de un arma y el poseerla puede reducir el miedo de su propietario. Los que tienen un arma por motivos de defensa, lo hacen como una respuesta individual a circunstancias de la vida colidiana percibidas como peligrosas. Sin embargo, la respuesta a estos peligros no está necesariamente mediada por el miedo; más bien parece ser parte de una menor preparación emocional frente a la posibilidad 
de un ataque (Kleck, 1991). Más aún, muchas de las personas que viven, o perciben vivir, en un ambiente hostil están convencidas de que su habilidad para sobrevivir depende de la forma en que vayan armados (ibid.).

Frenle a esta situación cabe hacer la pregunla, ¿qué tan eficaces son las armas de fuego como medio de defensa personal? Las respuestas a dicha inlenogante son complejas y se encuentran ampliamente divididas, sobre todo en Estados Unidos, entre criminólogos y especialistas en salud pública. Según Kleck (1991), uno de los máximos exponentes en investigación relacionada con armas de fuego, las personas que utilizan un arma para defensa propia corren menor riesgo de convertirse en víctimas frente a un crimen perpetrado, al no consumarse éste en forma completa; es decir, el crimen no es llevado a cabo en su totalidad -en un robo consistiría en que perdieran su patrimonio. Además, contrario a lo que comúnmente se cree, estas víclimas corren menor riesgo de ser lesionadas, en comparación con otras víctimas que usan otras formas de resistencia o que no ponen resistencia alguna (Kates et al., 1994). El mismo autor tampoco encontró evidencia que sustente la idea de que no oponer resistencia es menos peligroso que poner resistencia con un arma. El hecho de que las víctimas armadas puedan frustrar un crimen, sugiere que los civiles poseedores de amas de fuego pueden frenar a algunos delincuentes.

En la misma línea, John Lott Jr. (citado por TIME Magazine, 1998), economista de la Universidad de Chicago, publicó recienternente un libro titulado More Guns, Less Crimes: Undersianding Crime and Gun Control Laws [Más armas, menos delitos: entendiendo la delincuencia y las leyes de control de armas], el cual señala que la ciudadanía amada puede reducir la criminalidad. El estudio longitudinal realizado por Lott cubre un período de 18 años, de 1977 a 1995, y analiza los índices de criminalidad de 10 Estados que aprobaron leyes que autorizaban la posesión de armas. Según las cifras, el número de homicidios disminuyó en un promedio de $\mathbf{8}$ por ciento y los asaltos con violencia a un 7 por ciento. Además, de las 2.5 millones de veces que se empuñaron armas de fuego al año, en defensa propia, sólo en el 5 por ciento de los casos el portador resultó herido o lesionado. No obstante, dicho estudio, el más completo que se ha realizado según el autor en materia de armas, ha sido duramente criticado por defensores de las leyes que promueven el control de armas, ya que la conclusión a la que llegaría el estudio es que los ciudadanos que posean un arma pueden salvar su propia vida si se enfrentaran en algún ataque. Conclusión similar a la aportada por distintos criminólogos.

\subsection{Argumentos en contra}

En el otro extremo del debate de las armas se pueden encontrar numerosos argumentos en contra de ellas. Aun si se toman en cuenta los hallazgos de Kleck (1991), respecto al valor de las ammas en la defensa personal, el mismo autor 
menciona que las consecuencias de un desenlace fatal cuando se usa un ama de fuego son cuatro veces mayores que cuando se usan armas blancas -que son las siguientes armas más letales_ aunque se dieran las confrontaciones bajo las mismas circunstancías. Si la víctima tiene un arma, ésta puede defenderse, lo que puede - como ya se ha señalado- frenar a los posibles atacantes. Sin embargo, la facilidad de adquirir un amma también puede animar a los criminales a armarse primero (Roth, 1989), lo que podría acercarse más a la realidad salvadoreña. Según muestra la prensa salvadoreña, en los decomisos de armas que la Policía Nacional Civil realiza en operativos a bandas de delincuentes, se recolecta numeroso armamento, la mayoría de grueso calibre. Es más, según fuentes del Ministerio de Defensa, mensualmente son robadas alrededor de 100 armas, de las cuales sólo el 20 por ciento logra recuperarse (Funes et al., 1998).

Otro punto en contra es la imposibilidad de predecir cuándo un encuentro tendrá un desenlace con una lesión o una muerte. Esto debido a que no se pueden medir y controlar las intenciones, la intensidad del enojo del agresor y sus deseos de lastimar a la víctima en el momento del ataque (Kleck, 1991). Además, predecir la conducta violenta es difícil, en parte porque las investigaciones no han sido consistentes (Monahan, citado por Edwards, 1998). No obstante, se ha encontrado que la probabilidad de cometer actos violentos se incrementaba cuando había abuso de sustancias tóxicas (ibld.) y antecedentes criminales (Wintemute, 1998), dato que respalda las observaciones de conductas violentas en individuos que, entre otras características, usan drogas (Kellermann, 1996).

Por otro lado, numerosas investigaciones han demostrado que existe una obvia relación entre la posesión de armas de fuego, incidencia de ataques, defensas y muertes por arma de fuego (Kleck y Patterson, 1993; Guerrero et al., 1995; Roth, 1989; Kelleman, 1996; Kleck, 1991; Rand, 1994; Wintemute, 1998). Además, olros estudios en el exterior han demostrado que conductas como la portación de armas y el fácil acceso a ellas, están asociadas con la violencia y con diferentes conductas agresivas (Webster, Gainerand y Champion, 1993; Callahan y Rivera, 1992; Saltzman et al., 1992; Orpinas et al., 1998).

En tal sentido, Leonard Berkowitz, profesor de la Universidad de Stanford, Califomia (Tedeshi y Felson, 1995), y otros investigadores (Berkowitz y LePage, 1967; Frodi; 1975; Tumer y Simons, 1975; Turner, Simons, Berkowitz y Frodi, 1977, citados por Tedeshi y Felson, 1995), han realizado una extensa cantidad de experimentos para demostrar que las ammas tienen un efecto inhibidor o facilitador en la conducta agresiva. Berkowitz (ibid.) plantea que algunas señales de agresión funcionan como estímulos condicionados, los cuales pueden evocar respuestas agresivas. Según el referido autor, las armas funcionan como estímulos agresivos que permiten evocar conductas agresivas: "portar amas significa un incremento en la agresión: ellas crean agresión que en su ausencia no existiria" (Berkowitz, en Tedeshi y Felson, 1995, p. 61). 
Localmente existen diversas experiencias que muestran la incidencia de las armas en las tasas de homicidio. Específicamente, en Colombia, se pueden citar tres programas integrales destinados a mejorar la convivencia y la seguridad ciudadana, y a disminuir las tasas de homicidio. Dichos programas son: Consejería de Paz en Medellín, DESEPAZ en Cali y Convivencia en Bogotá (Hernández, 1998). Por citar uno de ellos, el programa DESEPAZ contemplaba una ley semiseca y la prohibición de porte armas, dentro de algunas de sus políticas. Además estaba constituido por cuatro áreas estratégicas: estudio sistemático de la violencia, fortalecimiento del orden institucional, educación para la paz y equidad y desarrollo social (Programa DESEPAZ, 1998). Según muestran los resultados desde que entró en vigencia el programa, en la ciudad de Cali, la tasa de homicidio ha disminuido hasta en un 20 por ciento (Guerrero et al., 1995).

\section{Contextualización del problema de las armas de fuego a la realidad sal- vadoreña}

Respecto a lo mencionado en los dos puntos anteriores es importante hacer algunas reflexiones para el contexlo salvadoreño. En primer lugar, muchas de las investigaciones que muestran el valor de las armas en la seguridad personal han sido realizadas en Estados Unidos de Norteamérica, donde los requisitos y las restricciones para obtener armas de fuego son diferentes y mucho más estrictas que las exigidas en el país. En segundo lugar, dentro de esas medidas se encuentra la división de licencia de porlación y tenencia de armas de fuego, lo que permite que no toda persona esté facultada para andar armada cuando lo desee. De hecho, aproximadamente, sólo el 5 por ciento de las personas que tienen un arma de fuego en Estados Unidos la portan (Kleck, 1991). Además, la mayoría de las armas en circulación son para uso recreacional o deportivo. Sólo un tercio del total de armas que circulan en dicho país son pistolas o revólveres (Wrigth, 1995).

Por otro lado, el panorama nacional de las armas de fuego se vislumbra diferente debido a múltiples factores históricos y coyunturales que el pais presenta. En primer lugar, la política de tenencia de armas es muy débil y distinta (para mayores detalles ver el apartado 5). Por otro parte, no hay que olvidar que Estados Unidos es un pais con una tasa de homicidios de 8.5 por cada 100,000 habitantes (McAlister et al., 1996); mientras que El Salvador llegó a presentar la tasa de homicidio más alta del continente con 138 por cada 100,000 habitantes 5 (Cruz y González, 1997, e Iglesias, 1997).

Con el fin de determinar empiricamente si las personas que tienen un arma de fuego sufren un grado de victimización menor que aquéllas que no poseen una, en el contexto salvadoreño, se elaboró una escala de victimización que aglutinaba seis reactivos contenidos en una investigación realizada por el IUDOP (1998), en la que se abordó el tema de las ammas de fuego $0^{h}$. A las personas se les 
planteó una serie de hechos deliclivos que pudieron haberles sucedido en el período de un año, a los cuales debían contestar cuántas veces les habían sucedido en el caso de haber sufrido alguno. Las respuestas con las cuales se elaboró la escala fueron dicotomizadas, de manera que éstas comprendían sólo dos condiciones: si les habla sucedido o no cualquiera de los hechos delictivos planteados. Los resultados demuestran que no existen diferencias en el grado de victimización entre las personas que poseen y no poseen un arma de fuego'; es decir, independientemente de que una persona tenga un arma de fuego o no, la probabilidad de que sea víctima de un hecho delincuencial es similar. Lo anterior desdice la tesis de que la tenencia de un ama de fuego reduce la probabilidad de que una persona sea víctima de un ataque o hecho delincuencial.

Otro aspecto que tiene un peso fundamental en la agenda nacional de las ammas es el aspecto cultural que le subyace. Especialistas confirman que dicho elemento, en las consecuencias sociales asociadas a las armas, es mucho más importante que mera disponibilidad de las ammas o el tipo de armas que pueden encontrarse circulando (Kates $e t$ al., 1994). La inclusión del aspecto cultural es importante debido a que el análisis de la violencia demanda ser estudiado tanto en términos cuantitalivos como, y en especial, cualitativos (Alvarenga, 1996). Además, dicho elemento puede conlrabalancear y definir algunas de las particularidades en el caso salvadoreño. En el país, a lo largo de muchas décadas, se ha desarrollado lo que algunos autores reconocen como la "cultura de la violencia". La "cultura de la violencia" privilegia el uso de comportamientos y creencias basadas en la efectividad de la agresión y la violencia para solucionar conflictos (Cruz, 1997b; Guenrero, 1997; Briceño-León et al., 1997; De Roux, 1997).

De hecho, algunos estudios parecen corroborar la existencia de tal patrón cultural. Por ejemplo, dos investigaciones realizadas en el Área Metropolitana de San Salvador (AMSS) (IUDOP, 1997; Beltrán et al., 1998), revelan que las personas que declaran tener armas de fuego tienen actitudes más favorables hacia acciones relacionadas con conductas violentas, que las personas que declaran no tener una. Una tercera investigación realizada con estudiantes del sistema educativo del AMSS (Cruz y Portillo, 1998), encontró que los estudiantes que portaron algún tipo de arma, presentaban mayores niveles de conducta agresiva que los estudiantes que no portaron ningún tipo de amas. Aún más, en un sondeo nacional realizado por el IUDOP (1998) se muestra que los salvadoreños que dicen poseer armas de fuego presentan un mayor grado de acuerdo frente a acciones ilegales, como ejercer la justicia por su propia cuenta si ésta no es proporcionada por el Estado, que las personas que declaran no tener una ${ }^{a}$.

Retomando la disquisición sobre la "cultura de la violencia", se puede decir que dicha dinámica fue reforzada en el período de la guerra civil, en la cual se vieron gravemente deterioradas las raíces de la convivencia social en El Salvador (Martín-Baró, 1990 y Cruz, 1997b). Sin embargo, el problema de la violen- 
cia en el país se puso de manifiesto históricamente mucho antes (Alvarenga, 1996 y Anderson, 1982).

El conflicto armado parece haber dejado secuelas que estimularon las condiciones para que se dé y se mantenga la violencia, como también un contexto posibilitador. Durante más de una década, los salvadoreños vivieron en una sociedad militarizada, donde la autoridad estaba claramente en manos de quienes poseían las armas. Muchos ciudadanos fueron adiestrados en el uso de la violencia como instrumento para poner fin a sus diferencias (Cruz,1997b).

La guerra mostró a los ciudadanos las ventajas del uso de la violencia para lograr los propósitos propios. A esto hay que sumarle todas las generaciones de salvadoreños que crecieron en este entorno de guerra y violencia, y que, por lo tanto, aprendieron la violencia como modo de vida. Aunque todos los ciudadanos hayan añorado la paz, muchos no sabían cómo actuar en una sociedad "pacífica", de manera que siguieron actuando como lo habían hecho durante muchos años. Aprendieron que un comportamiento agresivo podía ser válido y legítimo, aunque éste fuera contra las normas y las leyes establecidas (ibíd.).

En resumen, y en primer lugar, no se puede afimar a priori que la tenencia y uso de un arma por una víctima pueda reducir la posibilidad de que ésta sea atacada y la probabilidad de que el ataque tenga como resultado una lesión, por lo menos mientras no existan investigaciones más serias y profundas en el contexto salvadoreño. No obstante, desde una perspectiva térica, es ostensible sostener que un arma de fuego aumenta la probabilidad de que la lesión sea fatal, en el caso de que haya una. Además, dentro de este marco se hace necesario definir quién es la víctima y quién es el victimario, ya que estos papeles se pueden intercambiar entre un ladrón y un ciudadano común, una vez que un enfrentamiento armado tiene lugar. En segundo lugar, las investigaciones realizadas ponen de manifiesto que existe una clara relación entre armas de fuego y conducta agresiva, amas de fuego y tolerancia a la violencia, armas de fuego y "cultura de la violencia", y circulación de armas y tasas de homicidios. Finalmente, y en tercer lugar, para estudiar el impacto de las armas de fuego en el medio salvadoreño es indispensable incorporar condicionantes históricos, culturales y coyunturales propios, los cuales le dan sentido -desde una perspectiva nacional-al problema de las armas.

\section{Mercado y circulación de armas durante y después del conflicto armado}

Es un hecho que hasta ahora no se han realizado esfuerzos serios para determinar el impacto que está teniendo la armamentización de la población civil, en el período de la posguerra salvadoreña. Es más, nadie sabe a cabalidad cuántas armas de fuego se encuentran circulando en el país, ni tampoco en manos de quiénes están. Aunque las distintas oficinas de registro de armas tienen contabilizado el número de armas registradas hasta la fecha, éste no refleja -ni se 
acerca - la cifra real de las existentes. Aún más, las mismas autoridades del Ministerio de Defensa creen que existe una cantidad similar al número de armas registradas que se encuentran circulando ilegalmente. Sin embargo, es sólo un estimado de las fuentes militares (Funes et al., 1998).

El registro de armas comenzó a funcionar desde octubre de 1994, de manera que entre el período posterior a los acuerdos de paz y el período de operación de las oficinas de registro, no existía mayor control en la portación, tenencia, compra y venta de amas de fuego. Por si fuera poco, el conflicto bélico dejó un gran número de armas que quedaron circulando en manos de ex combatientes, de ambos bandos, y de civiles (Cruz, 1997b). Las cifras que se presentan a continuación, aunque no sean precisas - ni oficiales en algunos casos - dan un panorama del nivel de armamentización que predominaba durante y después de la guerra civil salvadoreña. Según un inventariado realizado por el FMLN (FMLN, sin año), la Fuerza Armada de El Salvador requisó entre los años de 1981 y 1991, en promedio, más de siete mil armas, de las cuales nunca se supo su paradero (ver Cuadro 1). Aunque los datos se basan en informes de prensa rendidos por la FAES, cabe la posibilidad de que ésta haya incautado muchas más armas de fuego a las fuerzas guerrilleras. Del lado de las fuerzas gubernamentales no se tiene conocimiento de la cantidad de armamento utilizado durante la guerra.

\begin{tabular}{lc}
\multicolumn{2}{c}{$\begin{array}{c}\text { Cuadro l } \\
\text { Armas del FMLN requisadas por la FAES } \\
\text { entre los años 1981-1991* }\end{array}$} \\
\hline Tipo de arma requisada & Totales generales \\
\hline Fusiles & 4,115 \\
Subametralladoras & 132 \\
Ametralladoras & 79 \\
Lanzagranadas & 363 \\
Morteros & 133 \\
Pistolas & 277 \\
\hline Total & 5,099 \\
\hline
\end{tabular}

Fuente: FMLN, sin año.

- No se incluyen municiones, minas, granadas y explosivos.

En el mismo proceso histórico, una vez finalizado el conflicto y firmados los acuerdos de paz, fue necesario operativizar acciones que comprendían la identificación, recolección y desirucción de ammamento y demás pertrechos de guerra, 
sobre todo de parte de los distintos grupos que se aglutinaron en el Frente Farabundo Martí para la Liberación Nacional (FMLN). Según un reporte elaborado por el Consejo de Seguridad de las Naciones Unidas sobre la misión de los Observadores de las Naciones Unidas en El Salvador (ONUSAL) (ONU, 1993, citado por Cruz, 1997b), el total de armas y municiones del FMLN identificadas desde el 1 de febrero de 1992, día en que entró en vigor el cese oficial de fuego, sobrepasaba el orden de las 10 mil, a parte de otros pertrechos de guerra. Posteriormente, dicha entidad llevó a cabo la recolección y destrucción de las armas del FMLN (ver Cuadro 2). No obstante, la cantidad de armas inventariadas después del tratado de paz nunca fue exacta según lo reportado (ONU, 1995, citado por Cruz, 1997b).

Además, como se mencionó anteriormenle, era de esperar que, una vez finalizada la guerra, una cantidad considerable de ex combatientes, tanto del FMLN como de la FAES, y de civiles mantuvieran en su poder amamento o pertrechos de guerra (Tojeira, 1997). De hecho, la realidad y las estadísticas actuales lo plantean de esa forma. Como afirma Cruz (1997b), los esfuerzos por recolectar el amamento disperso no fueron eficaces, ni tampoco rindieron los frutos esperados. En otro informe de Naciones Unidas (ONU, 1995, citado por Cruz, 1997b), a casi un año de la firma de los acuerdos de paz apenas se habían logrado recolectar 100 armas en poder de civiles, las cuales eran calculadas en miles.

\section{Cuadro 2 \\ Armas del FMLN destruidas por ONUSAL después de los acuerdos de paz*}

\begin{tabular}{lc}
\hline Tipo de arma destruida & Totales generales \\
\hline Armas individuales & 9,851 \\
Armas de apoyo & 379 \\
\hline Total & 10,230 \\
\hline
\end{tabular}

Fuente: Consejo de Seguridad de la ONU, 1993.

- No se incluyen municiones, minas, granadas, misiles, cohetes y explosivos.

Los esfuerzos para recolectar las armas fueron continuados en el período de posguerra, con el fin de reunir la mayor cantidad de armamento en manos de la población civil o desmovilizada. De esto se ha encargado el Movimiento Patriótico Contra la Delincuencia (MPCD), quienes reciben armas y pertrechos de guerra a cambio de bonos canjeables por bienes. Sin menospreciar la iniciativa 
llevada a cabo por dicho movimiento civil, ésta no ha redundado en grandes logros respecto al "desarme" de la sociedad salvadoreña. Desde septiembre de 1996 a julio de 1998, el MPCD ha recolectado más de tres mil armas de fuego, entre armas cortas y largas (ver Cuadro 3), con el apoyo y financiamiento del gobiemo salvadoreño y de otros países. Este esfuerzo significó una inversión de 9 millones de colones (Diario El Mundo, 1998).

\section{Cuadro 3}

Total de armas recolectadas* desde septiembre de 1996

a julio de 1998 en el programa de "bienes de consumo por armas de fuego" según el MPCD

\begin{tabular}{lcr}
\hline Año & Tipo de arma & Total \\
\hline 1996 & Corta & 718 \\
& Larga & 1,340 \\
1997 & Corta & 275 \\
& Larga & 744 \\
1998 & Corta & 112 \\
& Larga & 408 \\
\hline Subtotales & Corta & 1,105 \\
& Larga & 2,492 \\
\hline Torales & & 3,597 \\
\hline
\end{tabular}

Fuente: Elaboración propia basada en los registros del MPCD.

* No se incluyen explosivos, minas, misiles, cargadores, municiones y otros pertrechos de guerra.

A esta altura de la discusión es imporante apuntar que el conflicto armado no constiluye la única fuente de procedencia del amamento que actualmente circula entre la población civil. Según registros del Ministerio de Defensa, las armas que se encuentran matriculadas son poco más de 125 mil, entre armas cortas y largas (ver Cuadro 4). Sin embargo, las autoridades del Ministerio de Defensa calculan que existe una misma cantidad circulando ilegalmente (Funes et al., 1998). Por otro lado, Orellana Martínez (cilado por Tojeira, 1997) considera que hay alrededor de 250 mil amas ilegales en manos de civiles. Otras fuentes estiman que existen alrededor de ciento veinte mil armas no registradas, muchas por ser de uso privativo de la Fuerza Ammada. Además, según fuentes policiales, una buena cantidad de armas está en manos de menores de edad, lo que al final redundaría en un mayor número de personas amnadas de las que no 
se tiene conocimiento (Cruz, 1997b). Según un estudio realizado por el IUDOP (1996b), casi el 5 por ciento de los estudiantes de educación básica y media, con edades entre los 13 y los 18 años, del Área Metropolitana de San Salvador acudieron a recibir sus clases con armas de fuego. Otro dato que evidencia la gran cantidad de amas que circulan ilegalmente en el país es el número de delitos que se cometen con ese tipo de ammas: $\mathbf{8}$ de cada 10 delitos son cometidos por armas no matriculadas (Tojeira, 1997).

\section{Cuadro 4}

Total de armas matriculadas a nivel nacional desde octubre de 1994 a julio de 1998 según oficinas de registro de armas departamentales

\begin{tabular}{ccrrrrr}
\hline Año & Tipo de arma & San Salvador & Santa Ana & San Miguel & San Vicente & Total \\
\hline 1994 & Corla & 4,914 & 3,119 & 2,165 & 1,559 & 11,757 \\
& Larga & 874 & 1,203 & 358 & 267 & 2,702 \\
1995 & Corta & 20,605 & 9,924 & 11,634 & 6,250 & 48,413 \\
& Larga & 6,130 & 3,375 & 1,803 & 1,587 & 12,895 \\
1996 & Corta & 9,645 & 2,078 & 5,117 & 2,202 & 19,042 \\
& Larga & 3,170 & 671 & 863 & 470 & 5,174 \\
1997 & Corta & 8,189 & 1,428 & 4,607 & 1,229 & 15,453 \\
& Larga & 3,143 & 480 & 783 & 128 & 4,534 \\
1998 & Corta & 2,357 & 595 & 1,179 & 125 & 4,534 \\
& Larga & 767 & 191 & 272 & 7 & 1,237 \\
\hline \multirow{2}{*}{ Sub- } & Corta & 45,710 & 17,144 & 24,702 & 11,365 & 98,921 \\
totales & Larga & 14,084 & 5,920 & 4,079 & 2,459 & 26,542 \\
\hline Totales & & 59,794 & 23,064 & 28,781 & 13,824 & 125,463 \\
\hline
\end{tabular}

Fuente: Ministerio de Defensa.

Adicionalmente se cuentan con otros estudios que arrojan cifras de los niveles de armamentización de la sociedad salvadoreña. Según el Estudio ACTIVA (IUDOP, 1997), el 7 por ciento de la población del AMSS declaró tener un arma, lo que, según factores de expansión, significaría un promedio de 58,000 personas poseedoras de un arma de fuego. Datos oblenidos por el mismo instituto (IUDOP, 1998), en una encuesta realizada en junio del presente año, revelan que el 9 por ciento del total de la población salvadoreña declaró poseer un ama de fuego. Si los datos fueran probabilísticos, como los arrojados por el Estudio ACTIVA, se estaría hablando de más de $\mathbf{3 0 0}$ mil salvadoreños amados utilizando las proyecciones de la DIGESTYC (1996) para la mitad del presente año.

Aunque esta cifra es demasiado elevada, sugiere que el número de amas que se encuentran circulando es mucho más de lo que se ha dicho o se tiene conocimiento hasta el momento. Además, al parecer, por los datos aportados por el 
IUDOP, la cantidad de armas que se encuentran en circulación es mucho mayor en el interior del país: el $\mathbf{2 7 . 3}$ por ciento de los que declararon tener armas residen en el AMSS, mientras que el resto, 72.7 por cientc, vive en el interior del país. Sin embargo, el mayor registro de armas se efectúa en la zona metropolitana, según lo muestra el Cuadro 4. La discrepancia entre la distribución de armas en la zona metropolitana y el interior del país, con el número de matrículas realizadas en cada una de las cuatro oficinas de registro de armas, no puede explicarse por las cifras mismas de registro, ya que cualquier persona de otro departamento puede matricular su arma en San Salvador, así como en las otras oficinas departamentales. Por lo tanto, no puede determinarse mediante cifras oficiales la concentración de armas por zona geográfica.

Retomando los datos expuestos anteriormente y haciendo algunos cálculos sobre ellos se obtienen resultados no menos interesantes. Si se toma como cifra de referencia el número de armas destruidas por la ONUSAL $(10,230)$, recién terminado el conflicto bélico, se puede sostener que actualmente hay doce veces más armas circulando, por lo menos, legalmente. Las armas destruidas apenas representarían poco más del $\mathbf{8}$ por ciento de las armas que han sido registradas hasta la fecha $(125,463)$. Además, las armas recuperadas en perfodo de posgueпа, particularmente por el MPCD entre septiembre de 1996 y julio de 1998, apenas representan el 2.8 por ciento de las armas corlas y el 22.8 por ciento de las armas largas registradas en el mismo período".

A esto se le puede agregar que la demanda crea su propia oferta. Las armas, por lo menos las legales, representan un negocio de aproximadamente $165 \mathrm{mi}-$ llones de colones en el mercado (Funes et al., 1998). Esto quiere decir que el importe utilizado en programas para recuperar armas representa un marginal 5.5 por ciento de los dividendos que deja la venta de armas. No importa entonces que el acceso a las amas de fuego sea legal o ilegal. Mientras la demanda exista, siempre habrá quienes saquen provecho de estar abasteciendo el mercado.

Lo anterior pone de manifiesto que en el período de posguerra, la sociedad salvadoreña continúa presentando una alto nivel de armamentización, aunque no existe una idea clara y exacta del número de armas que se encuentran en circulación en la actualidad.

\section{Los vacíos de la ley de control de armas de fuego}

Parte del problema de las armas de fuego se explica por la misma ley de control de armas de fuego, municiones, explosivos y artículos similares vigente. La actual normativa que regula la extensión de permisos para la tenencia y portación de armas es lo suficientemente laxa para que casi cualquier persona pueda tener un arma de fuego, si así lo desea. Uno de los puntos más criticables de dicha normativa es la extensión de una única licencia, la cual no hace ninguna distinción entre la tenencia y la portación del arma. En segundo lugar, no 
establece con claridad los mecanismos mediante los cuales se constatarían algunas de las restricciones que prescribe la ley. Por ejemplo, ésta prohíbe la concesión de la licencia a "personas que consuman estupefacientes, drogadictos y alcohólicos" (Art. 46, literal c). Sin embargo, la ley no menciona cómo se comprobarán tales hábitos ni tampoco los crilerios que definirían a tales personas. Es más, aunque esté prohibida la tenencia y portación de armas de fuego a tales personas, no está penado portar armas en estado de ebriedad (Tojeira, 1997). En otro de los artículos (Art. 45) se prohibe la portación de armas de fuego en una serie de lugares, los cuales incluyen expendios de bebidas alcohólicas, salones, cervecerías u otros lugares similares. No obstante, tampoco se establecen medidas encaminadas a controlar el cumplimiento de tales restricciones.

Respecto a los requisitos que se exigen para obtener la licencia de portación y tenencia de arma, algunos fueron derogados por los diputados de la Asamblea Legislativa con el fin de que las personas registraran sus ammas (Rivera, 1997). Esto provocó que sólo en el año 1995 se registraran más de 60 mil armas de fuego. De hecho, ahora se exige únicamente la solvencia policial y un documento de identidad para tramitar la licencia en la oficina de registro de armas, de todo lo que la ley vigente estipula en el Artículo 20, literal $d$. Además, no se necesita pasar por evaluaciones psicofísicas, ni tener que aprobar ningún curso de manejo de armas de fuego.

La verdad es que registradas o no, las armas están allí y el registro legal no dice nada acerca de las razones del propietario en relación con el uso que desea darle a su arma. Además, la utilidad del registro de armas en la investigación del delito puede verse mermada por la falta de coordinación que existe entre la oficina de registro de armas, dependencia del Ministerio de Defensa, y la Policía Nacional Civil (Martínez, 1998).

La otra cara de la moneda, en el control de las armas, contiene el paquete de reformas que la Comisión de Defensa de la Asamblea Legislativa pretende implantar. De hecho, en el anteproyecto de reformas a la actual ley los diputados reconocen "[q]ue la falta de legislación adecuada que regule la posesión, tenencia y portación de armas de fuego, explosivos y accesorios, es un factor que ha contribuido al auge de la delincuencia en el país"'". Con base en esa y otras consideraciones, la ley vigente será modificada. Las reformas comprenderían una ampliación de los requisitos necesarios para registrar las armas de fuego y se establecería una división entre la licencia de tenencia y conducción, y la licencia de portación. Básicamente, la ley que entraría en vigencia comprendería los mismos requisilos, con la excepción de que se restablecería el requerimiento de la constancia de antecedentes penales (excluyendo delitos culposos) y se exigiría un curso y examen sobre el uso y manejo del arma, sólo para los que deseen obtener la licencia de portación. Las personas que posean la licencia de tenencia podrán acompañarse de su arma mientras ésta no vaya cargada. En 
cuanto a las restricciones, éstas se mantienen similares, aunque ahora se pedirá un examen médico para las personas que sufran limitaciones físicas y mentales. Las reformas, en general, mantienen lo dictaminado a la ley de control de armas vigente. La principal novedad de las reformas es la división de los tipos de licencia, como se mencionó anteriormente.

A pesar de que dichas reformas son adecuadas, no son del todo suficientes. Por ejemplo, se dejan de fuera evaluaciones psicológicas, las cuales son desestimadas debido a su falta de "objetividad", según lo expresado por las mismas autoridades vinculadas en la elaboración de las reformas (Funes et al., 1998). Adicionalmente, las penalizaciones de ciertos actos que atenten contra la vida, como realizar disparos al aire, deberían ser endurecidas.

Finalmente, es claro que la ley, aún cuando se incluyan nuevas reformas, no controlará completamente la libre circulación de armas en el país y las consecuencias que éstas acarrean. Para ello se tendría que fortalecer, en primer lugar, el sistema judicial y policial del país. Esto debido a que no todas las personas que cometen algún delito se les abre un debido proceso judicial y mucho menos reciben una penalización. Por ejemplo, de los 6,792 homicidios dolosos cometidos en 1996, según la Fiscalía General de República, sólo el 6 por ciento de los victimarịs fueron arrestados y sólo el 2.4 por ciento recibió una condena. Lo que quiere decir que el 94 por ciento del resto de homicidas podrian haber obtenido una licencia de portación o tenencia de un arma de fuego por no tener antecedentes penales. Por olro lado, se deberian establecer medidas que restrinjan factores asociados al uso de las armas, como lo son la ingesta de alcohol o el uso de drogas. Además, este control debería ser más estricto en períodos de temporada vacacional o de fiesta, ya que el número de muertes se incrementa, por ejemplo, en el mes de diciembre, principios de enero y otros períodos donde la ciudadanía goza de períodos feriados como se muestra en la Gráfica 1. Finalmente, se vuelven necesarias intervenciones "culturales", de concientización y campañas encaminadas a la resolución de conflictos, Jas cuales podrían complementar las medidas que permitan controlar el problema de las muertes violentas.

\section{Prevalencia de muertes por armas de fuego en el Área Metropolitana de San Salvador (AMSS)}

Los registros proporcionados por el Instituto de Medicina Legal y las alcaldías municipales muestran que, en el AMSS, una de las principales causas de muerte violenta es la muerte por arma de fuego. Aunque los datos que se presenIan a continuación corresponden esencialmente al AMSS, la prevalencia de las muertes y lesiones provocadas por arma de fuego parecen ser un fenómeno que se extiende al resto del país, según lo ponen de manifiesto el número de levantamiento de cadáveres y reconocimientos de sangre y sanidad realizados por el Instituto de Medicina Legal". 


\section{Gráfica 1}

Muertes por causa externa registradas mensualmente durante 1996 y 1997 en el AMSS según registros del Instituto de Medicina Legal

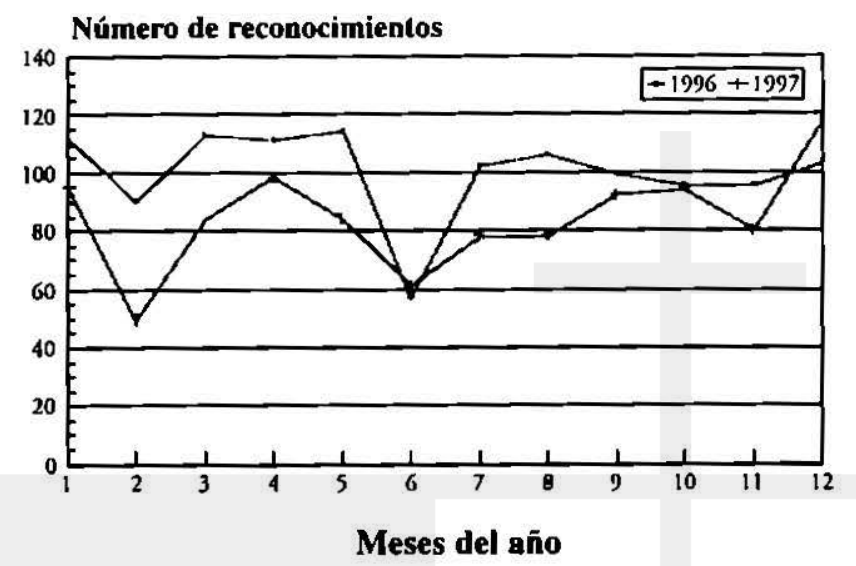

Fuente: elaboración propia con base en registros del IML.

En el año de 1995, según los registros del Instiluto de Medicina Legal, se contabilizaron 1,350 muertes por causas violentas sólo en el AMSS. Del total de muertes, el 50.2 por ciento fue causada por proyectil de arma de fuego según los datos del referido instiluto. En 1996, la cantidad de levantamientos realizados por el Instituto de Medicina Legal disminuyó a 1,197 muertes violentas; es decir, que se percibió una reducción del 12.4 por ciento en el número de muertes por causas violentas contabilizadas. No obstante, el porcentaje de muertes por arma de fuego fue similar: de las muertes registradas en 1996, el 49.7 por ciento correspondía a muertes producidas por lesión de algún tipo de arma de fuego. Si se observan los resultados de los municipios en forma separada, Apopa y San Salvador son los que acumulan el mayor número de muerles por arma de fuego (ver Cuadro 5). En estos dos municipios las muertes por arma de fuego representaron más de la mitad del total de muertes violentas acaecidas en dicho lugares; mientras que los demás municipios tienden a presentar porcentajes similares entre sí, con excepción de Ciudad Delgado y Ayutuxtepeque que presentaron porcentajes inferiores.

En 1997, la siluación de muertes por arma de fuego pareció agravarse, aún cuando el número de muertes violentas disminuyó sensiblemente en un 15.6 por ciento en el AMSS; es decir, de 1,197 muertes violentas registradas en 1996, hubo 1,010 registradas en 1997. Del total de muertes por causa externa en dicho año, el 55.2 por ciento correspondió a muertes por arma de fuego. Tomando 
como referencia el número de levantamientos de cadáveres efectuados en los años mencionados, se tendría que en 1997 se registraron 25 por ciento menos levantamientos de cadáveres que en 1995 . Un comportamiento similar, aunque no tan significativo, se puede observar en las tasas de victimización obtenidas en los últimos cuatro años, las cuales también han disminuido (IUDOP, 1998). En el Cuadro 5 se puede apreciar que en nueve de los diez municipios citados, los porcentajes de muertes por arma de fuego se han incrementado en relación con los porcentajes obtenidos para 1996.

\section{Cuadro 5}

Porcentaje de levantamientos de cadáveres por lesión de arma de fuego en el AMSS* según registros del Instituto de Medicina Legal durante 1996 y 1997

\begin{tabular}{lll}
\hline Municipio & 1996 & 1997 \\
\hline Apopa & 60.4 & 45.9 \\
Ayutuxtepeque & 19.4 & 57.1 \\
Ciudad Delgado & 37.5 & 44.4 \\
llopango & 42.4 & 67.1 \\
Mejicanos & 43.1 & 46.9 \\
San Marcos & 33.3 & 54.8 \\
San Martín & 44.4 & 55.2 \\
San Salvador & 54.5 & 56.7 \\
Santo Tomás & 42.9 & 60.0 \\
Soyapango & 46.4 & 48.5 \\
\hline
\end{tabular}

Fuente: elaboración propia a partir de los registros del IML.

- No se incluyen en los cálculos los municipios de Nucva San Salvador, Antiguo Cuscallán y Cuscatancingo para ambos años.

Lo anterior, además de indicar que las muertes por arma de fuego se encuentran en aumento, también apunta a que las muertes por causas externas se están especializando y concentrando hacia una sola: muertes producidas por arma de fuego. Esto cobra sentido si se analizan por separado las distintas causas registradas en el AMSS. Por ejemplo, las muertes producidas por proyectil de arma de fuego se han incrementado en un 5.5 por ciento de 1996 a 1997.

Por otro lado, el comportamiento de los resultados se plantea distinto para las muertes producidas por amma blanca, las cuales han disminuido en un 3.9 por ciento en el mismo período; mientras que las muertes causadas por artefactos explosivos se redujeron en un 1 por ciento de 1996 a 1997. Las otras causas de 
muerte violenta mencionadas - asfixia por ahorcamiento y politraumatismos-tienden a presentar cierta estabilidad en el tiempo. Los resultados se muestran en el Cuadro 6.

Como se puede apreciar, este primer acercamiento a la incidencia de las armas de fuego en la muerle de los capitalinos comienza a revelar una de las principales causas que se encuentran detrás de la mayor parte de homicidios o asesinatos.

\section{Cuadro 6}

Causas de muertes violentas en el AMSS* según registros del Instituto de Medicina Legal durante 1996 y 1997

\begin{tabular}{lrr}
\hline Causa de muerte & 1996 & 1997 \\
\hline Arma de fuego & 49.7 & 55.2 \\
Arma blanca & 16.5 & 12.6 \\
Artefacto explosivo & 2.1 & 1.1 \\
Asfixia por ahorcamiento & 7.2 & 7.0 \\
Politraumatizado & 20.4 & 22.7 \\
Indeterminada & 4.2 & 1.4 \\
\hline
\end{tabular}

Fuente: claboración propia a partir de los registros del IML.

- No se incluyen los municipios de Nueva San Salvador y

Antiguo Cuscallán.

Otra de las fuentes utilizadas para estudiar la incidencia de las armas de fuego en la morbilidad de la población capitalina son los registros de defunción de las alcaldías del AMSS, obtenidos directamente de los libros de defunciones anuales de cada una de ellas ${ }^{12}$. Según los registros de defunción, en 1995, las alcaldías reportaron un total de 1,460 defunciones por causas violentas ${ }^{13}$. (Cruz y González, 1997). De esas muertes, el 39.4 por ciento se vinculaba directamente con algún tipo de lesión provocada por arma de fuego. En 1996, según la misma fuente, el número total de muertes violentas registradas fue de 1,335 (ibid.). Del total de muertes, el 39.9 por ciento se debió a lesiones producidas por armas de fuego ${ }^{14}$. Según los registros de defunciones en los años 1995 y 1996, los porcentajes de muerte causada por armas de fuego se mantienen, prácticamente, constantes. Sólo en 1996 se da un pequeño incremento no significativo.

Si se comparan los porcentajes mostrados por los registros del Instituto de Medicina Legal y los recolectados por las alcaldías del AMSS, los datos de la primera fuente revelan un porcentaje mayor de muertes causadas por armas de fuego que la segunda. La diferencia entre ambas fuentes se encuentra en el orden del 10 al 15 por ciento. 
Respecto a lo anterior se pueden hacer varias anotaciones. En primer lugar, es necesario mencionar que los procedimientos mediante los cuales cada una de las fuentes registran las muertes son distintos. Las cifras del Instituto de Medicina Legal provienen de los reconocimientos de los cadáveres que realiza dicha institución; mientras que las cifras de las alcaldías están basadas en los registros de los libros anuales de partidas de defunción. En segundo lugar, las causas de muerte son catalogadas de distinta forma por cada una de las fuentes. En el caso del Instiluto de Medicina Legal, las causas se basan en expedientes médicos; mientras que en el caso de las alcaldías, la clasificación de la muerte muchas veces resulta poco clara o ambigua debido a que en muchos casos, nadie efectuó ningún reconocimiento del cadáver. Por tal razón se cuenta sólo con lo que declara el informante que acude a levantar el acta de defunción a la alcaldía.

En tal sentido, los expedientes que reportaron causas indeterminadas de muerte resultaron ser bastante altos en las alcaldías, llegando a representar casi el 25 por ciento de las muertes registradas ${ }^{15}$ (Cruz y González, 1997). Lo anterior podría explicar por qué en el Instituto de Medicina Legal el porcentaje de muertes violentas relacionadas con armas de fuego es mayor que en el de las alcaldías. Adicionalmente se puede mencionar que en muchas actas de defunción, la causa de la muerte era reportada más sobre efectos colaterales que por la causa directa que provocó la muerte (hemorragia, choque hipovolémico, trauma craneoencefálico, sepsis, elc.).

En otra línea, es claro que existe una segunda diferencia entre ambas fuentes: las alcaldías reportan mayor número de muertes violentas que las contabilizadas por el Instituto de Medicina Legal. Según los datos con los que se cuentan, las alcaldías del AMSS reportan poco más del 10 por ciento de muertes violentas que el Instituto de Medicina Legal. Cruz y González (1997) explicaban esta incongruencia por la forma en que el Instituto de Medicina Legal lleva sus registros, lo que genera un subregistro en la contabilización de las muertes. Funcionarios del referido instituto mencionaron que no todos los cuerpos de las personas que mueren en forma violenta pasan por la forensia, producto de la negativa de familiares para que se le practique la autopsia al cadáver. De esa forma, cerca del 30 por ciento de los casos son clasificados bajo "otras causas de muerte". En resumen, se puede decir que la forma en que efectúan el registro de muertes en ambas fuentes, permite que se dé un subregistro, ya sea en la causa en la que se cataloga la muerte o en la contabilización del número total de cadáveres con su respectiva causa de muerte.

Tomando en cuenta las limitantes de las fuentes citadas, ambas revelan resultados en los que la tasa de muertes por amma de fuego es elevada. Los datos muestran que cinco de cada diez muertes violentas en el AMSS, en 1995, 1996 y 1997, fueron causadas por lesión de armas de fuego. Un dato que no puede dejar de mencionarse respecto a las muertes por arma de fuego es que éstas, 
además de representar la principal causa de muerte violenta en el AMSS, se posicionan como la principal causa de muerte del total de levantamientos de cadáveres realizados en la zona geográfica en cuestión para los años 1995, 1996 y 1997 (IML, 1995; 1996 y 1997); es decir, las muertes provocadas por proyectil de arma de fuego no sólo constituyen la principal causa extema de muerte, sino que también son la principal causa de muerte, en general, en el AMSS según los registros del Instituto de Medicina Legal.

\section{Conclusiones}

No cabe duda que la armamentización de la sociedad civil es una respuesta al clima de inseguridad generado últimamente en el país. La mayor parte de las personas que oblienen un arma lo hacen para protegerse, según lo declaran ellas mismas. El flujo de ventas y el número de registros de armas de fuego en los últimos años pone de manifiesto que los salvadoreños se están apropiando de una parte de la protección que el Estado debe, y tiene la obligación, de proporcionar. Ahora que el gobierno cree conveniente realizar algunas reformas sobre la ley de control de armas, que anteriomente permilió que se conformara todo un ejército civil, para frenar el fácil acceso a las armas, hace falta establecer normas más estriclas que incluyan otros factores que están asociados a su uso. Aunque las personas tienen el derecho constitucional de armarse, éstas mismas están poniendo en entre dicho el concepto de seguridad ciudadana, el cual también está formando parte de la empresa privada. Es más, ese mismo derecho a armarse es el que legitima el comportamiento de la población, el cual a su vez permite cerrar el círculo de violencia interpersonal reforzado por paulas culturales. Esto debido a que participan como una pieza más que los califica como víctimas o viclimizadores potenciales en aras de la prolección personal.

Como menciona Wright (1995), las armas no son inherentemente buenas ni inherentemente malas; las armas no poseen teleologia. Lo bueno y lo malo reside en los motivos y la conducta de las personas, no en la tecnología que poseen. Un arma de fuego es un trozo de metal a la que puede dársele una gran variedad de usos. Lo Ilamamos bueno cuando el blanco para el cual se usó es el apropiado (ibid.). Las armas dan poder a los victimarios como a las víctimas cuando ellas responden en defensa propia. Como consecuencia, son fuente de orden y desorden social, dependiendo de quién las use, como sucede con el uso de la fuerza en general (Kleck, 1991). Por ello el control de la libre circulación de armas se vuelve necesario.

Por otra parte, desde una perspectiva teórica-práctica, diferentes estudios ponen de manifiesto la relación que existe entre armas de fuego, conducta agresiva, tolerancia a la violencia y tasas de homicidio. Además, diferentes autores consideran que el factor cultural es fundamental en la definición del problema subyacente a las armas de fuego. Y es ese factor, expresado en la "cultura de la 
violencia", el que le da un sentido distinto al caso salvadoreño, lo cual queda plasmado en la incidencia que tiene en las muertes causadas por arma de fuego.

Algunos de los resultados muestran un contraste sobre el que hay que llamar la atención, y es el que exisle entre los niveles de victimización y de homicidios, y la percepción de inseguridad por parte de los ciudadanos. Los datos indican que los índices de victimización y homicidios parecen estar reduciéndose en los últimos años mientras que el temor de la población sigue en aumento. Particularmente, las muertes por causa violenta en el AMSS se han reducido en una cuarta parte de 1995 a 1997; sin embargo, las muertes por armas de fuego, por el contrario, se han incrementado. Aunque no es posible delerminar las causas subyacentes a la disminución de la tasa de homicidios generales en el AMSS, lo que sí resulta claro es que las armas de fuego tienen un mayor impacto en las muertes acaecidas en dicha zona geográfica. Y aún en el interior del país. En forma adicional, el contraste anteriomente citado sugiere que el clima de inseguridad que prevalece actualmente se nutre más bien de otras fuentes distintas a la realidad misma o a una realidad "filltrada". Probablemente a cierlos grupos les interesa que este clima se siga respirando, ya que los dividendos que deja palear la angustia y el miedo de tantos son altos.

\section{Bibliografía}

Alvarenga, P., Cultura y ética de la violencia. El Salvador 1880-1932, San José: EDUCA, 1996.

Anderson, T., El Salvador 1932, San José: EDUCA, 1982.

Beltrán, M.A., Paganini, M. y Portillo, N.A., Actitudes hacia la violencia interpersonal y tenencia de armas de fuego, tesis para optar al grado de Licenciatura en Psicología, San Salvador: UCA, 1998.

Briceño-León, R., "Buscando explicaciones a la violencia", Revista Espacio Abierio, Vol. 6, No. 1, 1997b, pp. 45-67.

Callahan, C.M. y Rivera, F.P., "Urban High School Youth and Handguns”, JAMA, 267(22), 1992, pp. 3038-3042.

Cruz, J. M., "La cultura de la violencia como generadora de conflictos", Seminario taller, "Los medios de comunicación la prevención de conflictos y la cultura de Paz", San Salvador: UNESCO, El Salvador, 1997a, pp. 84-90.

Cruz, J. M., "Los factores posibilitadores y las expresiones de la violencia en los noventa", Estudios Centroamericanos (ECA), No. 588, San Salvador: UCA, El Salvador, 1997b, pp. 977-992.

Cruz, J. M. y González, L. A., "Magnilud de la violencia en El Salvador", Estudios Centroamericanos (ECA), No. 588, 1997, pp. 953-966.

Cruz, J. M. y Portillo, N., "Factores de riesgo asociados a la conducla agresiva en estudiantes de educación media del área de San Salvador", trabajo presentado en la conferencia "Violencia en una sociedad en transición", organizado por el PNUD, San Salvador, El Salvador (Mimeo), 1998.

De Roux, G. I., "Subdesarrollo, urbanización y violencia", Revisia Venezolana de Economia y Ciencias Sociales, Vol. 3, No. 2-3, 1997, pp. 145-162. 
Diario El Mundo, "Intercambio de bienes por armas", 13 de julio, 1998, p. 1.

DIGESTYC, Proyección de la población de El Salvador 1995-2025, San Salvador, EI Salvador, 1996.

Echeburúa, E., Personalidades violentas, Madrid: Pirámide, 1996.

Edwards, R., "Can Psychologists Predicl Violent Dehavior" en www.apa.org/monitor/ apr96/ignoreb.html, 1998.

El Diario de Hoy, "Las armas de fucgo en los Estados Unidos", Time Magazine 1 (15), 3 de julio, 1998, pp. 4-10.

FMLN, ¿Qué hizo el ejército con las 7 mil armas requisadas al FMLN?, (Mimeo), sin año.

Funes, M., Romero, C. y Segura, E, “Armas para todos", Enfoques, 1(11), pp. 1c-5c, en La Prensa Grafica, 16 de agosto, 1998.

González, L.A., "El Salvador en la postguerra: de la violencia armada a la violencia social", Revista de Ciencias Sociales y Humanidades, Realidad, No. 59, 1997, pp. 441-458.

Guerrero, R., "Plan de acción regional sobre violencia y salud", trabajo presentado en el Taller sobre "La violencia de los adolescentes y las pandillas ("maras") juveniles", San Salvador: Organización Panamericana de la Salud-OMS, 1997.

Guerrero, R.; McAlister, A.; Concha Eastman, A. y Espitia, V.E., Personal Disarment Deters Homicide in Cali, Colombia, (informe no publicado), 1995.

Hernández, R., "Apoyo a la convivencia y seguridad ciudadana", trabajo presentado en el Foro sobre "Convivencia y seguridad ciudadana en cl Isımo Centroaméricano, Haití y República Dominicana”, organizado por el BID: San Salvador, El Salvador (Mimeo), 1998.

Iglesias, E., "Los desafíos de la criminalidad urbana. Documento especial", Proceso, 17 (753), San Salvador: UCA, 1997, pp. 13-16.

Institulo de Medicina Legal, Anuario Estadístico, Corte Suprema de Justicia, San Salvador, El Salvador, 1997.

Instituto Intcramericano de Derechos Humanos, Seguridad ciudadana en Centroamérica, (Mimco), 1998.

Instituto Universitario de Opinión Pública, "La violencia en El Salvador", Estudios Ceniroamericanos (ECA), No. 569, 1996a, pp. 240-249.

Instituto Universitario de Opinión Pública, Estudio ACTIVA: "La Violencia en el Gran San Salvador", Boletín de prensa, Año XII, No. 5, 1997.

Instituto Universitario de Opinión Pública, "Los salvadoreños opinan sobre el problema de la delincuencia", Boletín de prensa, Año XIII, No. 3, 1998.

Instituto Universitario de Opinión Pública, "Sondeo sobre violencia en los centros educativos del área de San Salvador", Serie de Informes 59, $1996 \mathrm{~b}$.

Kates, D.B.; Schaffer, H.E.; Lattimer, J.K.; Murray, G.B. y Cassem, E.H., "Guns and Public Health: Epidemic of Violence or Pandemic of propaganda?", Tenesse Law Review, 61, 1994, pp. 513-596.

Kellermann, A., "Understanding and Preventing Violence: A Public Health Perspective", en www.ncjrs.org/pdffiles/prevviol.pdf, 1996.

Kleck, G., "Guns and Violence: A Summary of the Field", en www.cs.cmu.edu/ karl/ firearms/point-blank-summary.html, 1991.

Kleck, G. y Patterson, E.B., "The Impact of Gun Control and Gun Ownership Levels on Violence Rates", Journal of Quantitative Criminology, 9, 1993, pp. 249-287. 
Martin-Baró, I., Psicología Social de la Guerra, San Salvador: UCA Editores, 1990.

Martínez, M., "Controversia entre Defensa y PNC por registro de armas", El Diario de Hoy, 2 de agosto, 1998, pp. 8-9.

McAlister, A.; Velez, L.F.; de los Ríos, R. y Guencro, R., Culturals norms and attitudes toward violence in selected cities of the region of the Americas, Washington D.C.: Panamerican Health Organization (Mimco), 1996.

National Rifle Association-Institute for Legislative Aclion, "Compendium of State Laws Governing Fircarms", en www.nra.org/gun-law/compend.pdf, 1997.

Organización de las Naciones Unidas, "Informe del Secrelario de las Naciones Unidas sobre la misión de los obscrvadores de las Naciones Unidas en El Salvador (ONUSAL)", dirigido al Conscjo de Seguridad el 30 de agosto de 1993 (Mimeo).

Orpinas, P.; Murray, N. y Kelder, S., Parentals influences on students' aggressive behaviors and weapon-carrying (Mimco), 1998.

Polsby, D. y Brennen, D., "Taking Aim at Gun Control", en www.hearlland.org/geps.htm, 1995.

Procuraduría para la Defensa de los Derechos Humanos, ONUSAL, Gula sobre las normas y procedimientos de la Policia Nacional Civil, San Salvador, El Salvador, i 994.

Programa DESEPAZ, "Un esfuerzo integral para combatir la violencia urbana en Cali, Colombia", trabajo presentado en el Foro sobre Convivencia y scguridad ciudadana en el Isımo Centroaméricano, Haití y República Dominicana, organizado por el BID: San Salvador, El Salvador (Mimeo), 1998.

Rand, M., "Guns and Crime; Handgun Victimization, Fircarm Self-defense and Firearm Theft", en www.ncjrs.org/uxfiles/gunc.uxt, 1994.

Rivera, C., "Violencia instilucionalizada en El Salvador", La Prensa Grafica, San Salvador, El Salvador, 16 de junio, 1997, pp. 4-5,

Roth, J. A., "Fircarms and Violence", en www.ncjrs.org/tutfiles/fireviol.txt, 1989.

Rubio, M., "Los costos de la violencia en América Lalina", Irabajo presentado en el Foro sobre Convivencia y seguridad ciudadana en el Istmo Centroaméricano, Haití y Repúb́lica Dominicana, organizado por el BID: San Salvador, El Salvador (Mimeo), 1998.

Saltzman, L.E.; Mercy, J.A.; O'Carroll, P. W.; Rosenberg, M.L. y Rhodes, P. H., “Weapon Involvement and Injury Outcomes in Families and Intimate Assaults", JAMA, 267(22), 1992, pp. 3043-3047.

Tedeshi, J. T. y Felson, R. B., Violence, aggression and coercive actions, Washington D.C.: American Psychological Association, 1995.

Tojeira, J. M., "El problema de las amas", Carta a las iglesins, XVI, No. 389, 1997, pp. 8-9.

Websters, D.W.; Gainer, P. S. y Champion, H. R., "Weapon Carrying Among Inner-city Junior High School Students: Defensive Behavior vs. Aggressive Delinquency", American Journal of Public Health, 83(11), 1993, pp. 1604-1608.

Winlemute, G., "Predicting Criminal Behavior Among Authorized Purcharsers of Handgungs", en www.ncjrs.org/pdffiles/fs000198.pdf, 1998.

Wright, J., "Ten Essential Observations on Guns in America", en www.assc.org/afc/ afclenob.html, 1995.

\section{Notas}

1. Analista ll del Instituto Universitario de Opinión Pública (IUDOP).

2. Este artículo incluye apartados contenidos en el trabajo de graduación "Actifudes hacia la violencia interpersonal y tenencia de armas de fuego", realizado por 
Antonieta Beltrán, Michel Paganini y Nelson Portillo para optar al grado de Licenciatura en Psicología.

3. Los dalos corresponden a una investigación realizada por el Instituto Universitario de Opinión Pública (IUDOP) de la UCA y financiada por el Banco Iniermericano de Desarrollo (BID) durantc 1997.

4. Aunque existen requisitos generales, cada Estado puede regularlos de distinta forma. Para más detalles ver, por cjemplo, National Rifle Association-Institute for Lcgislative Action, 1997.

5. El Salvador, desde los años setenta, acusaba un elevado índice de mucries por violencia intencional, ubicándose en el primer lugar en las estadísticas regionales. La tasa de muertes por homicidios era alrededor de 30 por cada 100 mil habilantes, mientras que en Colombia y Brasil no eran mayores a 25 muertes por cada 100 mil habitantes (Cruz, 1997a).

6. Los reactivos incluidos en la escala son: (1) jalguien le robó a mano armada en los últimos 12 meses?; (2) ¿alguien le amenazó para sacarle o pedirle dinero en los últimos 12 meses?; (3) ¿fue usted golpeado por otra u otras personas en los últimos 12 meses?; (4) ¿fue usted herido con un arma blanca en los últimos 12 meses?; (5) ¿fue usted herido con un arma de fuego en los últimos 12 meses? y (6) ien los últimos 12 meses, lola amenazaron de muerle a usted o a algún pariente cercano?

7. $\times 2=0.11746, p=0.14563$.

8. $F=6.5, p=0.01$.

9. El porcentaje correspondiente a armas largas es mucho más alto, debido a que la mayor parte de armas entregadas fueron armas de guerra.

10. Terecra consideración de la Asamblea Legislativa contenida en el anteproyecto de reformas a la Ley de Control de Armas de Fuego, Municiones, Explosivos y Arlículos similares.

11. Según datos oficiales del Instituto de Medicina Legal, el 56.7 por ciento de las muertes violentas identificadas en 1995, en el territorio nacional, sucedieron por lesión provocada por arma de fuego; mientras que en 1997, el 51.5 por ciento de las muertes violentas de la región Paracentral se dieron por la misma razón. La manera en que los datos son presentados en el Anuario Estadístico del referido instituto no permite determinar el porcentaje de muertes por arma de fucgo en las regiones Occidental y Oriental del país.

12. No incluye los municipios de Santo Tomás y Cuscatancingo.

13. Excluyendo las muertes violentas accidentales.

14. Este porcentaje excluye lodas las muertes provocadas por artefactos explosivos para ambos años.

15. Debido a que el porcentaje de muertes indeterminadas era demasiado alto, Cruz y González aplicaron un análisis discriminante para determinar el número de muertes que podrían deberse a causas violentas. El análisis determino que, para 1995, el 15 por ciento de muertes indelerminadas se pueden atribuir a hechos violenlos; mientras que para 1996, el porcentaje estimado fue del 14 por ciento. De esta forma, las cifras totales incluyen tales registros. 